\title{
Efficacy and safety of pulsatile gonadotropin-releasing hormone therapy in patients with congenital hypogonadotropic hypogonadism: a multicentre clinical study
}

\author{
Ming Hao ${ }^{1,2 \#}$, Jiang-Feng Mao ${ }^{1 \#}$, Qing-Bo Guan ${ }^{3}$, Long Tian ${ }^{4}$, Hu Han ${ }^{4}$, Hong-En Lei ${ }^{4}$, \\ Dong-Mei Zheng ${ }^{3}$, Zhen-Hua Tian ${ }^{3}$, Min Nie $^{1}$, Xi Wang ${ }^{1}$, Bing-Qing Yu ${ }^{1}$, Yin-Jie Gao ${ }^{1}$, Xue-Yan Wu ${ }^{1}$ \\ ${ }^{1}$ NHC Key Laboratory of Endocrinology, Peking Union Medical College Hospital; Department of Endocrinology, Peking Union Medical College \\ Hospital, Peking Union Medical College, Chinese Academy of Medical Sciences, Beijing, China; ${ }^{2}$ Department of Endocrinology, The First Affiliated \\ Hospital of Harbin Medical University, Harbin, China; ${ }^{3}$ Department of Endocrinology, Shandong Provincial Hospital Affiliated to Shandong \\ University, Jinan, China; ${ }^{4}$ Department of Urology, Beijing Chao Yang Hospital, Beijing, China \\ Contributions: (I) Conception and design: XY Wu; (II) Administrative support: L Tian, QB Guan, XY Wu; (III) Provision of study materials or \\ patients: JF Mao, X Wang, DM Zheng, ZH Tian, H Han, HE Lei; (IV) Collection and assembly of data: M Nie, BQ Yu, YJ Gao, M Hao; (V) Data \\ analysis and interpretation: M Hao; (VI) Manuscript writing: All authors; (VII) Final approval of manuscript: All authors. \\ \#These authors contributed equally to this work. \\ Correspondence to: Xue-Yan Wu. NHC Key Laboratory of Endocrinology, Peking Union Medical College Hospital; Department of Endocrinology, \\ Peking Union Medical College Hospital, Peking Union Medical College, Chinese Academy of Medical Sciences, Beijing 100730, China. \\ Email: sheyanwu@vip.sina.com.
}

Background: Pulsatile gonadotropin-releasing hormone (GnRH) therapy may restore function of the hypothalamus-pituitary-gonad axis and induce spermatogenesis in male patients with congenital hypogonadotropic hypogonadism $(\mathrm{CHH})$. The study sought to test the reliability of a newly developed Innopump ${ }^{\circledR}$ hormone pump, and to confirm the efficacy and safety of pulsatile GnRH therapy (by Innopump ${ }^{\circledR}$ hormone pump) in $\mathrm{CHH}$ patients.

Methods: From November 2017 to November 2018, 28 male patients with CHH were treated with pulsatile GnRH at Peking Union Medical College Hospital, Beijing Chaoyang Hospital, and Shandong Provincial Hospital. A prospective, self-controlled, 7-day clinical trial was conducted. The primary outcome measures were the efficacy and safety of pulsatile GnRH therapy (which was administered via the Innopump ${ }^{\circledR}$ hormone pump). The secondary outcome measures included total serum testosterone, luteinizing hormone (LH) and follicle stimulating hormone (FSH) levels.

Results: All of the patients participated the clinical study. For 7 days, a dosage prescribed by doctors was accurately administered by the Innopump ${ }^{\circledR}$ hormone pump, and recorded by the pump. During the treatment, LH and FSH levels gradually increased to 2.66 \pm 1.74 and 5.05 $\pm 3.03 \mathrm{IU} / \mathrm{L}$, respectively. Upper respiratory tract infection in 1 patient and slight nausea in another patient were reported, which were confirmed to be unrelated to the pulsatile GnRH therapy.

Conclusions: The Innopump ${ }^{\circledR}$ hormone pump was found to be reliable in drug administration, and to have an accurate alarming system. It effectively and safely treated patients with CHH. Pulsatile GnRH therapy may produce a physiological pattern of $\mathrm{GnRH}$ secretion, and re-establish pituitary-gonad axis function by increasing gonadotropin levels.

Keywords: Pulsatile gonadotropin-releasing hormone therapy (pulsatile GnRH therapy); Innopump ${ }^{\circledR}$ hormone pump; congenital hypogonadotropic hypogonadism $(\mathrm{CHH})$; spermatogenesis

Submitted Feb 10, 2021. Accepted for publication Jun 16, 2021.

doi: $10.21037 /$ atm-21-1632

View this article at: https://dx.doi.org/10.21037/atm-21-1632 


\section{Introduction}

Transition from childhood to adulthood, also called pubertal development, relies on the activation of the hypothalamicpituitary-gonadal (HPG) axis $(1,2)$. Coordinated neuroendocrine action may produce pulsatile gonadotropinreleasing hormone $(\mathrm{GnRH})$ secretion with periodicity and amplitude. Under physiological conditions, the pulsatile release of $\mathrm{GnRH}$ from the hypothalamus during sleep is a marker of pubertal initiation. The periodicity and amplitude of the pulsatile rhythm of GnRH then gradually mature, producing the luteinizing hormone $(\mathrm{LH})$ and follicle stimulating hormone (FSH) from the pituitary gland. Defects in GnRH synthesis and action cause delayed pubertal development, hypogonadism and infertility (1).

Congenital hypogonadotropic hypogonadism $(\mathrm{CHH})$ is a disorder of the hypothalamus, which leads to decreased GnRH secretion, followed by low levels of LH and FSH, and finally gonadal hypoplasia. The main therapeutic ways to induce spermatogenesis are pulsatile GnRH therapy, and combined human chorionic gonadotropin (HCG) and human menopausal gonadotropin (HMG) therapy. GnRH can be subcutaneously administered by a portable and minimal pump at intervals of 60-90 minutes. GnRH pump may produce a physiological model for spermatogenesis in patients with $\mathrm{CHH}$ (3). Compared to combined HCG/ HMG therapy, pulsatile GnRH therapy may produce earlier spermatogenesis, a higher pregnancy rate, and a normal testosterone level.

The pulsatile GnRH hormone pump is also referred to as an artificial hypothalamus, as it can produce pulsatile GnRH to induce pituitary gonadotropin secretion. The instrument is precisely controlled by a microcomputer, and pulsatile GnRH is subcutaneously administered. Dosage and frequency can be adjusted individually. The calculated injections are accurate and simple. The portable pump is also easy to carry around. Thus, the GnRH pump is recommended as the preferred therapy for the long-term treatment of patients with $\mathrm{CHH}$.

In this study, the reliability of a newly developed portable Innopump ${ }^{\circledR}$ hormone pump was examined. The effectiveness and safety of pulsatile GnRH therapy in patients with $\mathrm{CHH}$ were also evaluated. We present the following article in accordance with the TREND reporting checklist (available at https://dx.doi.org/10.21037/atm-211632).

\section{Methods}

\section{Patients}

In this longitudinal, prospective, multicentre, and selfcontrolled clinical study (ChiCTR1900027378), 28 patients with $\mathrm{CHH}$ were recruited. The participants were patients at the Peking Union Medical College Hospital, Beijing Chao Yang Hospital, and Shandong Provincial Hospital from November 2017 to November 2018. To be eligible to participate in the study, the patients had to meet the following inclusion criteria: (I) be aged between $16-40$ years old; (II) have serum LH and FSH levels below 1 IU/L, and low sex hormone levels; and (III) have discontinued HCG, HMG, and sex hormonal therapy for at least 4 weeks. Conversely, patients were excluded from the study if they met the following exclusion criteria: (I) had cryptorchidism or testosterone of $<1.0 \mathrm{ng} / \mathrm{mL}$ after a HCG stimulating test; (II) have multiple anterior pituitary hormone deficiency caused by hypothalamic pituitary lesions; (III) have hypergonadotropic hypogonadism; and/or (IV) have psychosis, an allergy to GnRH, or any other systemic disease, such as chronic obstructive pulmonary disease or chronic kidney failure.

Each patient's clinical presentations, medical history, and family history were recorded on the patient's first visit to hospital. The Ethics Committee of the Peking Union Medical College Hospital, Beijing, China granted approval for the study (No. JS-2111). Informed consent was obtained from each patient after a full explanation of the purpose of this study had been provided. All procedures performed in this study involving human participants were in accordance with the Declaration of Helsinki (as revised in 2013).

\section{Treatment and follow-up}

Pulsatile GnRH (Fengyuan Pharmaceutical Co., Anhui Province, China) was administered subcutaneously via a portable hormone pump (LenoMed Coropartion, Shanghai, China) for 7 days. Gonadotropins and sex hormones (i.e., $\mathrm{LH}, \mathrm{FSH}$, testosterone, and testicular size) were measured at baseline, day 4 , and day 7. Compliance and adherence to the Innopump ${ }^{\circledR}$ hormone pump was assessed at each visit by a participant report. For each patient, a GnRH dosage was initiated at $10 \mathrm{ug}$ per 90 minutes (4).

After 7 days of pulsatile GnRH therapy, the patients could elect 1 of the following 3 methods to continue their 
Table 1 Baseline features of patients with congenital hypogonadotropic hypogonadism ( $\mathrm{n}=28$ )

\begin{tabular}{lcc}
\hline Baseline characteristics & All patients $(\mathrm{n}=28)$ & Reference range \\
\hline Age (year), mean \pm s.d. & $24.23 \pm 4.63$ & - \\
Height $(\mathrm{cm})$, mean \pm s.d. & $175.08 \pm 6.88$ & - \\
Weight $(\mathrm{kg})$, mean \pm s.d. & $74.43 \pm 16.16$ & - \\
Basal T (ng/mL) & $0.37(0.28,0.58)$ & $1.75-7.81$ \\
Basal LH (IU/L), median (quartiles) & $0.20(0.20,0.28)$ & $1.24-8.62$ \\
Basal FSH (IU/L), median (quartiles) & $0.53(0.31,0.86)$ & $2.27-9.26$ \\
\hline
\end{tabular}

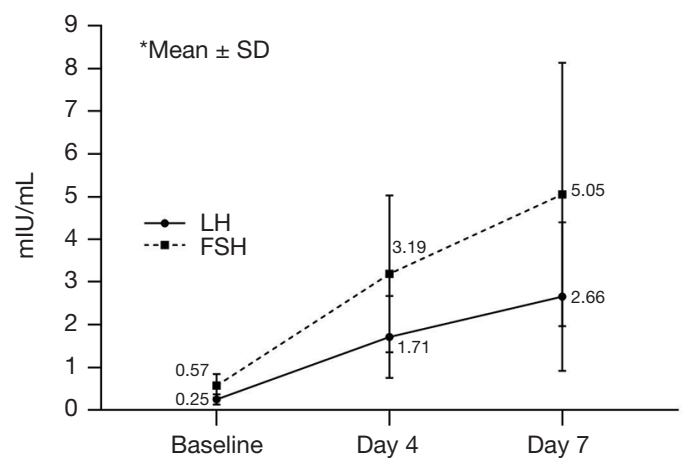

Figure 1 Patients' FSH and LH levels increased gradually after 1 week of pulsatile GnRH pump therapy ( $\mathrm{n}=28)$. LH, luteinizing hormone; FSH, follicle stimulating hormone.

therapy: (I) pulsatile GnRH; (II) combined HCG/HMG treatment; or (III) testosterone supplementation. Regular follow-up examinations were conducted at intervals of 3 months. Each patient's testicular size, plasma gonadotropins, plasma testosterone, and sperm count were measured on each visit.

The mini pump used in this study has been approved by the State Food and Drug Administration and the Shanghai Supervision and Inspection Centre for Medical Device Quality.

\section{Statistical analysis}

SPSS version 17.0 (SPSS Inc., Chicago, IL, USA) was used for the data analysis. Normal distributive data are expressed as the mean \pm standard deviation (SD). Nonnormal distributive data are listed as medians (quartiles). Sample-paired $t$-tests were used to compare changes in gonadotropins and testosterone levels at baseline and after treatment. Statistical significance was set at $\mathrm{P}<0.05$.

\section{Results}

\section{Patient demographics}

A total of 28 male patients were recruited to participate in the study, comprising 16 patients from Peking Union Medical College Hospital, 6 patients from Beijing Chaoyang Hospital Affiliated to Capital Medical University, and 6 patients from Shandong Provincial Hospital. The patients were all in good health and had normal blood and urine routine test results, and normal liver and renal function. Thyroid hormone, adrenal glucocorticoid, and growth hormone levels were all within normal ranges.

The patients were $23.24 \pm 4.63$ years in age, and had a body mass index of $24.6 \pm 3.8 \mathrm{~kg} / \mathrm{m}^{2}$. Baseline serum $\mathrm{LH}$, $\mathrm{FSH}$, and testosterone levels were $0.20(0.20,0.28) \mathrm{IU} / \mathrm{L}$, $0.53(0.31,0.86) \mathrm{IU} / \mathrm{L}$, and $0.37(0.28,0.58) \mathrm{ng} / \mathrm{mL}$, respectively (see Table 1).

\section{Accurate injection and reliability of the mini pump}

Over the 7-day treatment period, a total of 1,645 injections were administered, and recorded by the pump. The dosage and frequency were completely consistent with that required by the doctors, indicating that the pump had a high accuracy and reliability for GnRH injections.

\section{Gonadotropin and sex hormone levels post-treatment}

Patients' levels of FSH and LH significantly increased after treatment. On the 4th day of treatment, FSH and LH levels increased to $3.19 \pm 1.84$ and $1.71 \pm 0.96 \mathrm{IU} / \mathrm{L}$, respectively. On the 7th day of treatment, FSH and LH levels increased to $5.05 \pm 3.03$ and $2.66 \pm 1.74 \mathrm{IU} / \mathrm{L}$, respectively (see Figure 1). The rate of pituitary response to pulsatile $\mathrm{GnRH}$ was $100 \%$. 

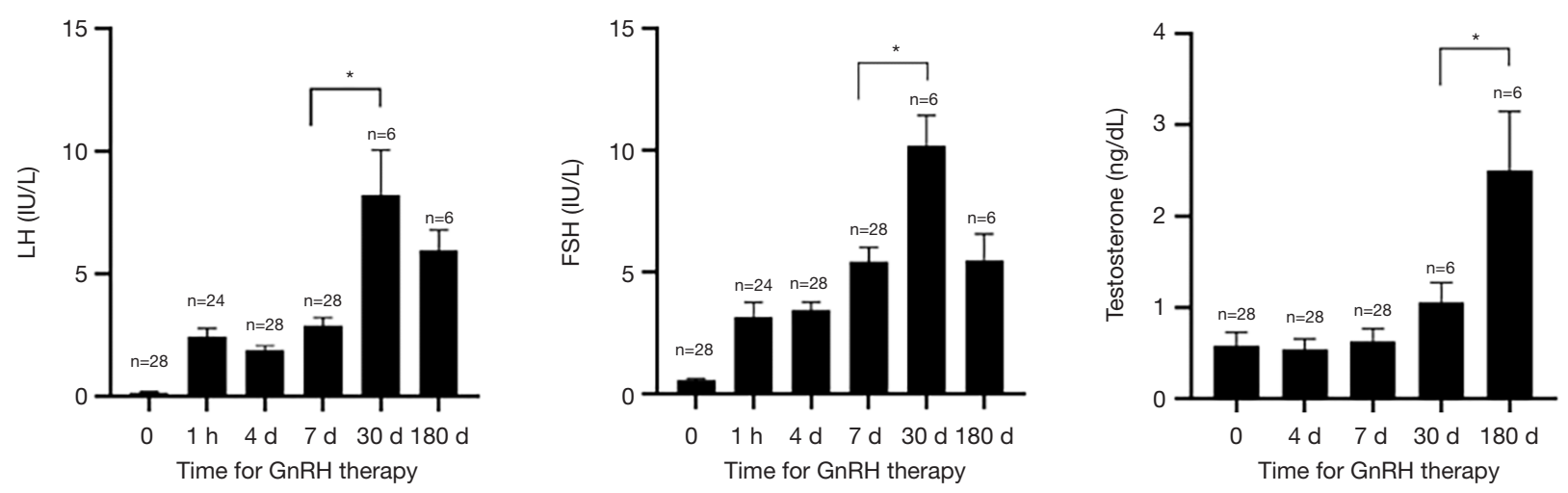

Figure 2 Patients' levels of gonadotropin and testosterone increased gradually during pulsatile GnRH therapy. A star $\left(^{*}\right)$ indicates that the differences between the 2 groups were statistically significant $\left({ }^{*} \mathrm{P}<0.05\right)$. LH, luteinizing hormone; FSH, follicle stimulating hormone; $\mathrm{GnRH}$, gonadotropin-releasing hormone.

\section{Safety of the pulsatile GnRH pump and adverse reactions of patients}

Over the 7-day treatment period, the pumps' alarms sounded 21 times. Of these alarms, 19 alarms occurred due to power exhaustion, 1 due to pipeline blockage, and 1 due drug to exhaustion. All of the alarms were effective. Thus, the pump has an alarm validity of $100 \%$.

Two adverse events were reported; 1 patient reported an upper respiratory tract infection, and another patient reported mild stomach discomfort. No serious adverse events occurred. No patients withdrew from the clinical study due to adverse events.

\section{Therapeutic preferences at the end of the trial}

When the 7-day clinical trial was completed, the patients could elect which treatment they wished to receive next. Seven patients elected to receive sex hormonal replacement therapy due to their were not seeking fertility treatment, 13 patients elected to receive HCG/HMG therapy, and 10 patients elected to continue to receive pulsatile $\mathrm{GnRH}$ therapy.

Six of the 10 patients who continued to receive pulsatile GnRH therapy were regularly followed up for more than 6 months. The LH and FSH levels of these patients gradually increased. After 1 month, their FSH and LH levels plateaued (see Figure 2). Six months later, patients' LH levels increased from $0.16 \pm 0.20$ to $5.96 \pm 2.04 \mathrm{IU} / \mathrm{L}$, and their FSH levels increased from $0.56 \pm 0.30$ to $5.51 \pm 2.60 \mathrm{IU} / \mathrm{L}$. Their serum total testosterone increased from $0.58 \pm 0.82$ to $2.50 \pm 1.59 \mathrm{ng} / \mathrm{mL}$. However, it should be noted that patients' serum testosterone levels did not increase until one month after treatment. It then slowly rose to the normal adult level in half a year. Seminal tests showed that sperm was detected in 1 out of 6 patients after 6 months of treatment, and the success rate of spermatogenesis was $16.67 \%$.

\section{Discussion}

In 1978, it was first demonstrated that GnRH was secreted in the form of pulses (5). Some 4 years later, in 1982, 6 $\mathrm{CHH}$ male patients were treated with intravenous injections of pulsatile GnRH, and spermatogenesis occurred in 3 patients, which confirmed that exogenous pulsatile GnRH may restore pituitary gonadal axis function in patients with $\mathrm{CHH}$ (6). However, at that time, the injection instruments were too large to be carried with the patients. However, with developments in technology and equipment, portable pumps have appeared. Such pumps can regularly deliver pulsatile GnRH at intervals of 90-120 minutes, which may mimic the physiological state of GnRH secretion. It has been proven that compared to HCG/HMG therapy, pulsatile GnRH may induce a larger testicular volume (7-9), require a shorter period for spermatogenesis, and have a better spermatogenesis success rate $(4,9,10)$. Thus, pulsatile GnRH therapy is the preferred therapeutic choice for male $\mathrm{CHH}$ patients who wish to father children (11).

This clinical trial showed that this newly developed Innopump ${ }^{\circledR}$ hormone pump is extremely accurate and highly safe at administering pulsatile GnRH infusions. The drug dosage and infusion frequency was completely 
consistent with the dosage prescribed by the doctors. The alarm system was $100 \%$ effective, and no false or invalid alarms occurred in the study. During the treatment, no technical problems were reported, such as electric shocks, the falling off of pumps or other apparatus, or infusion set leakage. Thus, this portable pump is both high quality and highly convenient. Additionally, no patients withdrew from the clinical study due to difficulties in manipulating the pump, which suggests that the operating system is easy to use for patients. In addition to being efficient, safe, and cost effective, the Innopump ${ }^{\circledR}$ hormone pump has also innovatively adopted advanced technology to enhance its convenience for users. For the first time, an Organic Light Emitting Diode color screen with a large display and touch function was adopted, which improved visualization and made the pump easier to use. Additionally, the Innopump ${ }^{\circledR}$ hormone pump is the only pump equipped with Bluetooth, which can be used to transfer treatment information to mobile phones and the "cloud," and provides an important basis for the network of disease management in various hospitals.

During the treatment period, patients' FSH and LH levels gradually increased, revealing a good pituitary response to pulsatile $\mathrm{GnRH}$ therapy. In relation to gonadotropin changes in response to pulsatile $\mathrm{GnRH}$ therapy, the present study showed that: (I) for most patients, levels of gonadotropin gradually increase within 1 week of treatment, which reflects a favorable response to GnRH therapy; (II) the increments of LH and FSH during the first 7 days of treatment can be used to predict the therapeutic effects of long-term pulsatile GnRH; (III) patients' testosterone levels rose relatively slowly after approximately 1-3 months of treatment, and may reach a stable plateau after 6 months; (IV) patients who attended the long-term follow-up examinations showed that pulse GnRH treatment was effective for a long period; however, their drug resistance could not be observed; and (V) patients who respond well to the therapy may produce sperm after 6 months of treatment.

Previous retrospective studies have shown changes in $\mathrm{LH}, \mathrm{FSH}$, and testosterone levels after pulsatile $\mathrm{GnRH}$ treatment. However, consistent with previous clinical studies $(12,13)$, this prospective study showed that FSH and LH levels plateaued after one month. This study also showed that patients' testosterone levels did not increase until 30 days after treatment. Thus, during pulsatile GnRH treatment, clinicians should be aware that low testosterone levels after 1 week of treatment may occur to ensure they do not incorrectly judge these results as a treatment failure (14). A slowly increasing testosterone level is similar to normal pubertal development. When boys enter puberty, GnRH pulse is initially secreted, after which, testicular volume and testosterone level gradually increase. Thus, it is generally advisable for clinicians to observe patients for 3-6 months when evaluating the therapeutic effect of pulsatile $\mathrm{GnRH}$ therapy.

Two mild adverse events were recorded in the study. After careful investigated, no relationship between these events and the drug therapy could be determined. No other adverse reactions, such as erythema at the infusion site, were reported.

The study had a number of limitations. First, as a prospective study, it was not designed to be randomized or double-blind. It is a self-control observational study. Second, the number of patients receiving long-term pulsatile GnRH therapy was too small and the observation time was too short to evaluate the final spermatogenesis effect.

In conclusion, the Innopump ${ }^{\circledR}$ hormone pump is accurate, effective, and safe in the treatment of $\mathrm{CHH}$. More than 1 month is required to determine the therapeutic effect of GnRH treatment in $\mathrm{CHH}$ patients. Increases in gonadotropins, testosterone, and testicular volume during the first 3-6 months of treatment are favorable indicators for predicting future spermatogenesis.

\section{Acknowledgments}

Funding: The study was funded by the National Key Research and Development Program of China (No. 2016YFC0905102, and No. 2016YFA0101003), the CAMS Innovation Fund for Medical Sciences (CIFMS) (No. 2016I2M-1-002, and No. 2017-I2M-3-007), and the Project of National Natural Science Foundation of China (No. 81771576).

\section{Footnote}

Reporting Checklist: The authors have completed the TREND reporting checklist. Available at https://dx.doi. org/10.21037/atm-21-1632

Data Sharing Statement: Available at https://dx.doi. org/10.21037/atm-21-1632

Conflicts of Interest: All authors have completed the ICMJE 
uniform disclosure form (available at https://dx.doi. org/10.21037/atm-21-1632). The authors have no conflicts of interest to declare.

Ethical Statement: The authors are accountable for all aspects of the work in ensuring that questions related to the accuracy or integrity of any part of the work are appropriately investigated and resolved. All procedures performed in this study involving human participants were in accordance with the Declaration of Helsinki (as revised in 2013). The Ethics Committee of the Peking Union Medical College Hospital, Beijing, China granted approval for the study (No. JS-2111). Informed consent was obtained from each patient after a full explanation of the purpose of this study had been provided.

Open Access Statement: This is an Open Access article distributed in accordance with the Creative Commons Attribution-NonCommercial-NoDerivs 4.0 International License (CC BY-NC-ND 4.0), which permits the noncommercial replication and distribution of the article with the strict proviso that no changes or edits are made and the original work is properly cited (including links to both the formal publication through the relevant DOI and the license). See: https://creativecommons.org/licenses/by-nc-nd/4.0/.

\section{References}

1. Foster DL, Jackson LM, Padmanabhan V. Programming of GnRH feedback controls timing puberty and adult reproductive activity. Mol Cell Endocrinol 2006;254255:109-19.

2. Choi JH, Yoo HW. Control of puberty: genetics, endocrinology, and environment. Curr Opin Endocrinol Diabetes Obes 2013;20:62-8.

3. Rastrelli G, Corona G, Mannucci E, et al. Factors affecting spermatogenesis upon gonadotropin-replacement therapy: a meta-analytic study. Andrology 2014;2:794-808.

4. Mao JF, Liu ZX, Nie M, et al. Pulsatile gonadotropinreleasing hormone therapy is associated with earlier spermatogenesis compared to combined gonadotropin therapy in patients with congenital hypogonadotropic hypogonadism. Asian J Androl 2017;19:680-5.

5. Belchetz PE, Plant TM, Nakai Y, et al. Hypophysial responses to continuous and intermittent delivery of hypopthalamic gonadotropin-releasing hormone. Science 1978;202:631-3.

6. Hoffman AR, Crowley WF Jr. Induction of puberty in men by long-term pulsatile administration of lowdose gonadotropin-releasing hormone. N Engl J Med 1982;307:1237-41.

7. Gong C, Liu Y, Qin M, et al. Pulsatile GnRH Is Superior to hCG in Therapeutic Efficacy in Adolescent Boys With Hypogonadotropic Hypogonadodism. J Clin Endocrinol Metab 2015;100:2793-9.

8. Liu L, Banks SM, Barnes KM, et al. Two-year comparison of testicular responses to pulsatile gonadotropin-releasing hormone and exogenous gonadotropins from the inception of therapy in men with isolated hypogonadotropic hypogonadism. J Clin Endocrinol Metab 1988;67:1140-5.

9. Schopohl J, Mehltretter G, von Zumbusch R, et al. Comparison of gonadotropin-releasing hormone and gonadotropin therapy in male patients with idiopathic hypothalamic hypogonadism. Fertil Steril 1991;56:1143-50.

10. Huang B, Mao J, Xu H, et al. Spermatogenesis of pulsatile gonadotropin-releasing hormone infusion versus gonadotropin therapy in male idiopathic hypogonadotropic hypogonadism. Zhonghua Yi Xue Za Zhi 2015;95:1568-71.

11. Boehm U, Bouloux PM, Dattani MT, et al. Expert consensus document: European Consensus Statement on congenital hypogonadotropic hypogonadism-pathogenesis, diagnosis and treatment. Nat Rev Endocrinol 2015;11:547-64.

12. Mao JF, Wang X, Zheng JJ, et al. Predictive factors for pituitary response to pulsatile GnRH therapy in patients with congenital hypogonadotropic hypogonadism. Asian J Androl 2018;20:319-23.

13. Hughes VA, Boepple PA, Crowley WF Jr, et al. Interplay between dose and frequency of GnRH administration in determining pituitary gonadotropin responsiveness. Neuroendocrinology 2008;87:142-50.

14. Pitteloud N, Thambundit A, Dwyer AA, et al. Role of seminiferous tubular development in determining the FSH versus LH responsiveness to GnRH in early sexual maturation. Neuroendocrinology 2009;90:260-8.

(English Language Editor: L. Huleatt)

Cite this article as: Hao M, Mao JF, Guan QB, Tian L, Han H, Lei HE, Zheng DM, Tian ZH, Nie M, Wang X, Yu BQ, Gao YJ, Wu XY. Efficacy and safety of pulsatile gonadotropinreleasing hormone therapy in patients with congenital hypogonadotropic hypogonadism: a multicentre clinical study. Ann Transl Med 2021;9(12):962. doi: 10.21037/atm-21-1632 